\title{
Study of the Spectral Properties of Nanocomposites with CdSe Quantum Dots in a Wide Range of Low Temperatures
}

\author{
K.A. Magaryan ${ }^{1 a}$, I.Y. Eremchev ${ }^{2}$, K.R. Karimullin ${ }^{1,2}$, M.V. Knyazev ${ }^{2}$, M.A. Mikhailov ${ }^{1}$, I.A. Vasilieva ${ }^{1}$, \\ and G.V. Klimusheva ${ }^{3}$ \\ ${ }^{1}$ Moscow Pedagogical State University, 29 Malaya Pirogovskaya str., 119992 Moscow, Russia \\ ${ }^{2}$ Institute for Spectroscopy, Russian Academy of Science, 5 Fizicheskaya str., 142190 Moscow, Troitsk, Russia \\ ${ }^{3}$ Institute of Physics, NAS of Ukraine, 46 Prospect Nauki, Kiev, 03028, Ukraine
}

\begin{abstract}
Luminescence spectra of the colloidal solution of CdSe quantum dots (in toluene) were studied in a wide range of low temperatures. Samples were synthesized in the liquid crystal matrix of cadmium octanoate $\left(\mathrm{CdC}_{8}\right)$. A comparative analysis of the obtained data with previous results was performed.
\end{abstract}

Keywords: optical spectroscopy, CdSe nanocrystals, quantum dots, polycrystalline powder, nanocomposites.

The optical properties of quantum dots depend essentially on the synthesis conditions. Use of liquid crystal materials for the synthesis of the quantum dots is characterized by number of advantages, for instance: possibility to control the size and shape of the nanocrystals at the stage of growth, to control their orientation in the liquid phase at high temperature [1]. In addition to the use of liquid crystals as host medium for the synthesis of the quantum dots, they are also interesting as new optical nanocomposite materials because they can combine properties of semiconductor heterostructures and the liquid crystal anisotropic medium [2]. One of the representatives of the ionic liquid crystals is metal alkanoate. CdSe quantum dots were grown inside the liquid crystalline phase of cadmium octanoate $\left(\mathrm{CdC}_{8}\right)$ that was used as a template for the synthesis of our samples.

The sample was synthesized in the ionic thermotropic liquid crystal matrix of cadmium octanoate [3]. Liquid crystal mesophase crystallizes by rapid cooling to the room temperature, forming glassy nanocomposite with nanoparticles. Polycrystalline powder with CdSe quantum dots inside (which is the object of this study) can be obtained by slow cooling, when the process of synthesis of the nanoparticles is finished.

Figure 1 (left hand spectrum) shows two absorption curves. The dotted line is related to the glassy nanocomposite with 2,3 $\mathrm{nm}$ CdSe quantum dots that was registered earlier [4] (see the description of the experimental technique in $[5,6])$. The solid line refers to the polycrystalline powder of cadmium octanoate with CdSe quantum dots (dissolved in toluene). As can be seen from the figure, all absorption maxima of the two curves coincide, except for the one peak at 460,5 $\mathrm{nm}$. On the basis of the band overlapping we can conclude that the size of quantum dots inside the glassy nanocomposite and inside the solution with polycrystalline powder is the same.

\footnotetext{
${ }^{a}$ Corresponding author: xmagaros@gmail.com
} 

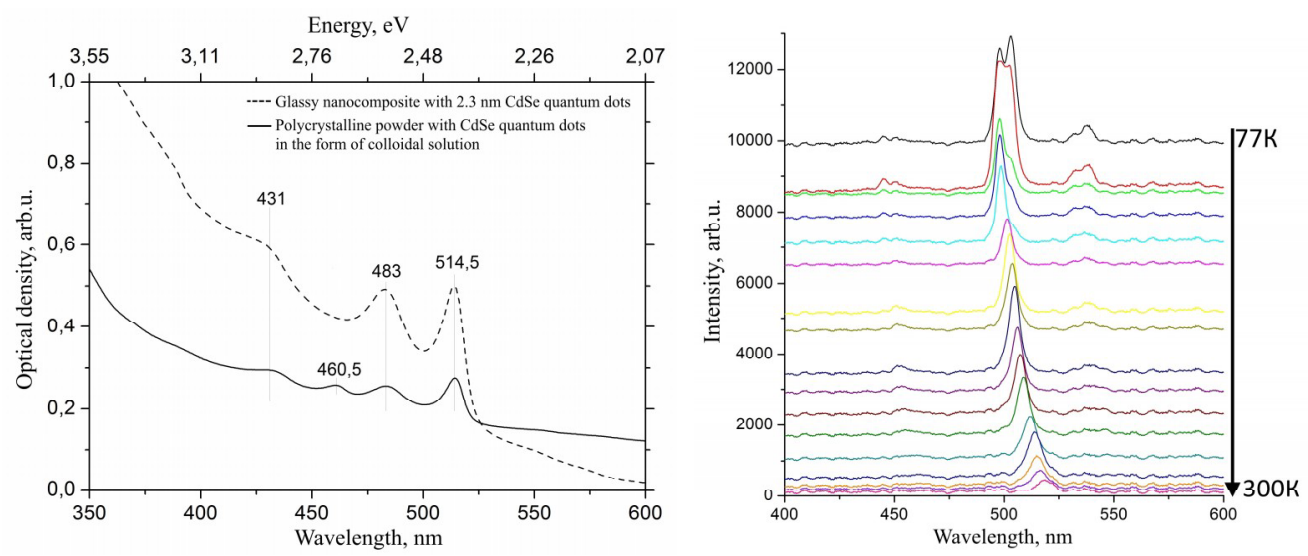

Figure 1. Absorption spectra of the samples. The dashed line shows the absorption curve for glassy nanocomposite with $2,3 \mathrm{~nm}$ CdSe quantum dots. The solid line is for polycrystalline powder with CdSe quantum dots in the form of colloidal solution in toluene (on the left). Fluorescence spectra of polycrystalline powder solution with CdSe quantum dots at temperatures ranging from $77,3 \mathrm{~K}$ to $300 \mathrm{~K}$ (on the right).

The luminescence spectra in the temperature range from $77,3 \mathrm{~K}$ to $300 \mathrm{~K}$ are shown on the right side of Figure 1. It demonstrates the relationship between exciton maximum position and temperature. Similar experiment was performed for glassy nanocomposites in [4]. Appearance of the second exciton peak as the temperature falls is a distinctive feature of these spectra. This peak appears when temperature lowers close to the liquid nitrogen boiling point, and its position is almost identical to the position of the first peak. The luminescence spectrum of glassy nanocomposite with the same size quantum dots shows similar two-humped exciton maxima. However, the intensity of both peaks is practically the same, and the distance between them is much greater. In the red region of the spectrum there is a band that does not depend on the temperature. The same band was observed earlier and it can be corresponded to the exciton trapping on the defects of nanocrystals. The intensity of this band is substantially smaller comparing to the one of the glassy nanocomposite.

For a more comprehensive study of the optical spectra dynamics of the objects it is necessary to reduce the concentration of quantum dots in a solvent in order to detect single nanocrystals. Such investigations are of the great interest both for the fundamental science and for developing the superresolved fluorescence microscopy [7-9].

We thank prof. T. Mirnaya from V.I. Vernadskii Institute of General and Inorganic Chemistry, National Academy of Sciences, Kyiv, Ukraine for providing us with the samples. We also thank prof. A. Naumov from Institute for Spectroscopy RAS for consultations and discussions of the results.

The work is supported by the Russian Foundation for Basic Researches (project No 15-32-21100). K. Magaryan acknowledges the Ministry of Education and Science of the Russian Federation (project No 14.B25.31.0007).

\section{References}

1. Y. Zhang et al., ACS Nano 9, 3. P. 3097-3108 (2015)

2. T.A. Mirnaya and S.V. Volkov Green Industrial Applications of Ionic Liquids (Eds. R. D. Rogers et al.) (Kluwer Academic Publ., P. 439, 2002)

3. G. Klimusheva et al., Liq. Cryst. 40, No. 7, P. 980-988 (2013)

4. K.A. Magaryan et al., Bull. Russ. Acad. Sci.: Phys. 78, No. 12, P. 1336-1340 (2014)

5. N.L. Naumova et al., Opt. Spectr. 92, 426 (2002); Opt. Spectr. 98, 535 (2005)

6. N.L. Naumova et al., J. Lumin. 111, 37 (2005).

7. A.V. Naumov, Physics Uspekhi. 56. P.605 (2014)

8. A.V. Naumov et al., Eur. Phys. J. D 68. P. 348 (2014)

9. I.S.Osad'ko, I.Yu.Eremchev, A.V.Naumov, J. Phys. Chem. C (2015) in press. 